\title{
Perfluorinated Self-Assembled Monolayers Enhance the Stability and Efficiency of Inverted Perovskite Solar Cells
}

Christian M. Wolff ${ }^{\text {A* }}$, Laura Canil ${ }^{\mathrm{Ba}}$, Carolin Rehermann ${ }^{\mathrm{Bc}}$, Nguyen Ngoc Linh ${ }^{\mathrm{C}}$, Fengshuo Zu E,F, Maryline Ralaiarisoa ${ }^{\mathrm{E}}$, Pietro Caprioglio A,Bb, Lukas Fiedler A, Martin Stolterfoht A, Sergio Kogikoski Jr. A, Ilko Bald ${ }^{A}$, Norbert Koch E,F, Eva L.Unger Bc,G, Thomas Dittrich $^{\mathrm{B}}$, Antonio Abate ${ }^{\mathrm{Ba}, \mathrm{D}}{ }^{*}$ and Dieter Neher ${ }^{\mathrm{A}}$

A. Universität Potsdam

Karl-Liebknecht-Str. 24-25

14776 Potsdam, Germany

B. Helmholtz-Zentrum Berlin für Materialien und Energie

Kekuléstraße 5

12489 Berlin, Germany

a. Young Investigator Group Active Materials and Interfaces for Stable Perovskite Solar Cells

b. Young Investigator Group Perovskite Tandem Solar Cells

c. Young Investigator Group Hybrid Materials Formation and Scaling

C. Institute for Molecular Engineering

The University of Chicago, Chicago

Illinois 60637, USA

D. Department of Chemical, Materials and Production Engineering University of Naples Federico II

Piazzale Tecchio 80, 80125 Fuorigrotta, Naples, Italy

E. Institut für Physik \& IRIS Adlershof, Humboldt-Universität zu Berlin, 12489 Berlin, Germany

F. Helmholtz-Zentrum Berlin für Materialien und Energie GmbH, 12489 Berlin, Germany

G. Department of Chemistry and NanoLund, Lund University, 22100 Lund, Sweden 


\section{Supplementary Note 1}

\section{Modelling the monolayer I-PFC12 on the perovskite surface.}

We calculated I-PFC12 on perovskite surface with a fully trans-conformation. Calculations were performed using the generalized gradient approximation (GGA) by Perdew-BurkeErnzerhof ${ }^{1}$ for the exchange and correlation functional, and adding van der Waals interaction corrections $^{2}$, accounting for the non- covalent interactions between the molecules and the surface. The electron-ion interactions were computed using ultrasoft pseudopotentials, including scalar relativistic effects. The cut-off energy for the plane-wave expansion was 30 Ry (300 Ry for the charge density cutoff). Brillouin-zone integration was performed with the special-point $^{3}$ done with a $6 \times 6 \times 1$ Monkhorst-Pack grid. The semiconductor surface was modelled employing a periodically repeated slab geometry using the $(1 \times 1)$ supercell with adsorbates on one side of the slab only (see Scheme below). The slabs were five layers thick, and the bottom layers were kept fixed in their bulk positions during relaxations. A $12 \AA$ vacuum layer was used, which was found to be sufficient to ensure negligible coupling between periodic replicas of the slab. All calculations were performed using the PWscf code contained in the Quantum-ESPRESSO distribution ${ }^{4}$ Molecule-molecule interaction was neglected and the conformational freedom of the perfluorocarbon chain was restricted. The calculation predicts values of 2.103 and $2.921 \AA$ for the distances of the carbon-iodine bond in the molecule and the nucleophilic iodine-electrophilic iodine, respectively. These are bond lengths that are close to the values reported for the halogen bonds of I-PFC12 (intramolecular iodine-carbon) on the silicon nitride (iodine-nitride) surfaces41.

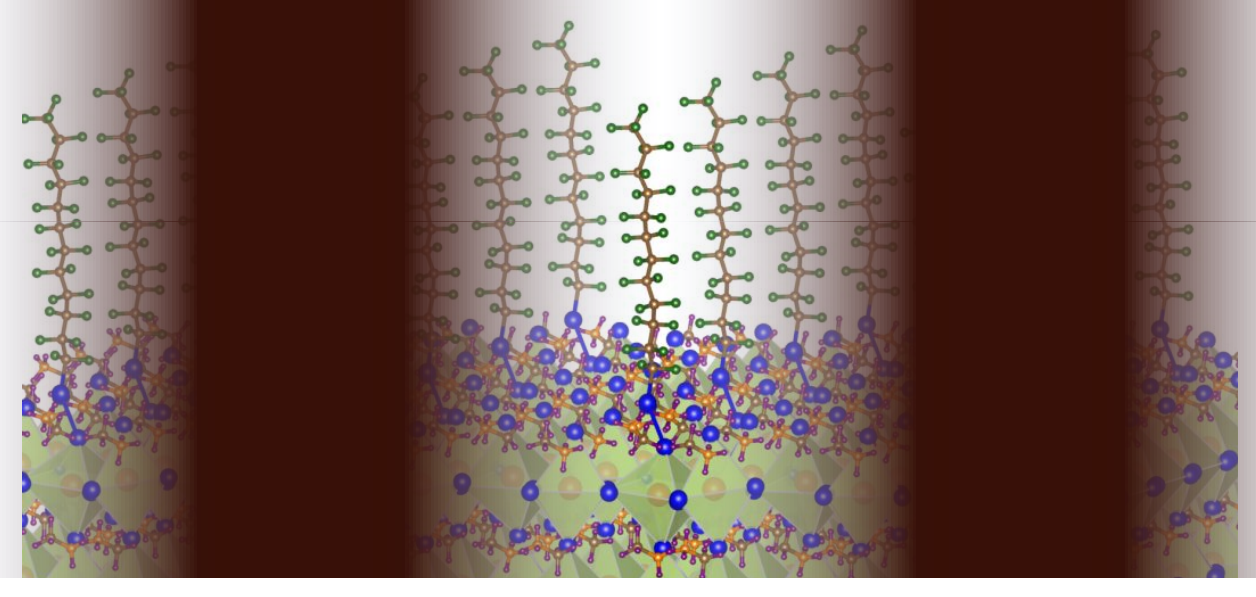


Scheme S1: Density functional theory (DFT) simulations of multiple I-PFC12 molecule and a perovskite (Iterminated)

\section{Supplementary Note 2}

\section{Details on reconstruction of $\mathrm{V}_{\mathrm{OC}}$ / QFLS}

\section{$\mathrm{EQE}_{P V} \& \mathrm{EQE}_{\mathrm{EL}}$}

Detailed balance invokes that any material that absorbs radiation is likewise emitting radiation simply due to fact that it functions as a black body emitter (BB) at a finite temperature. The equivalent current of emitted photons in $\mathrm{A} / \mathrm{m} 2$ is then calculated with

$J_{0, \text { rad }}=q \cdot \phi_{\text {emission }, 0}=q \cdot \int E Q E_{P V}(E) \cdot \phi_{B B}(E) d E$

While the absorbed current can be obtained by convoluting the $\mathrm{EQE}_{\mathrm{PV}}$ with the spectrum of the light source used (in this case a solar simulator mimicking AM1.5G)

$J_{S C}=\int E Q E_{P V}(E) \cdot \phi_{S u n}(E) d E$

In the case, where the luminescent efficiency of the device under forward bias is known - $\mathrm{EQE}_{\mathrm{EL}}$ - the $V_{\mathrm{OC}}$ can then be calculated with

$V_{O C}=\left(k_{B} \cdot T\right) / q \cdot \ln \left[E Q E_{E L} \cdot J_{S C} / J_{0, \text { rad }}+1\right]$.

We show the EQE spectra with and without SAMs in Figure S2b.

\section{PLQY \& QFLS}

Alternative to injecting current into a device - which requires the fabrication of a full device in the first place - charge carriers can also be generated by illuminating a sample with light. The photoluminescence intensity is directly linked to the quasi-Fermi level splitting (QFLS, $\Delta E_{F}$ ) via

$\ln \left(\frac{I_{P L}(E) h^{3} c^{2}}{2 \pi E^{2}}\right)=-\frac{E}{k T}+\frac{\Delta E_{F}}{k T}$.

$\Delta E_{F} / q$ is then a direct measure of the maximum obtainable open circuit voltage in a device or a stack. This model however is highly sensitive to variations in the PL spectra, as the high energy tail is used to determine the temperature of the emitting species. This can render temperatures that are unrealistically high. The origin of this behavior is not yet understood. Therefore for the herein shown values we used formula $\mathrm{S} 3$ but exchange $E Q E_{\mathrm{EL}}$ by the external photoluminescence yield PLQY at charge carrier concentrations equivalent to $J_{\mathrm{SC}} /\left(d^{*} q\right)$, essentially by calibrating the illumination source such, that if we measure a device we achieve 1-sun $J_{\mathrm{SC}}$. This can be done under the assumption that the IQE of the perovskite devices is constant and close to $100 \%$, i.e. photogeneration or injection can be exchanged. 


\section{Time-resolved PL}

Generally the Shockley-Queisser equation can be solved for $\mathrm{J}=0$ to:

$V_{O C}=\epsilon_{G}+\frac{k_{B} T}{\cdot} \cdot \ln \left(\frac{n_{e} w_{h}}{N_{C} N_{V}}\right)$

Thus under the premise of knowing the charge carrier densities, $V_{\mathrm{OC}}$ can be constructed while keeping in mind, that at open-circuit generation $=$ recombination, i.e.

$\frac{d n_{\theta}}{d t}=G-R=0 \sim G-\frac{n_{\Theta}}{\tau_{S R H}} \rightarrow n_{\Theta} \sim G \tau_{S R h}$

Here $n_{e / k}$ the density of electrons/holes in the conduction/valence band, $G$ is the generation rate,

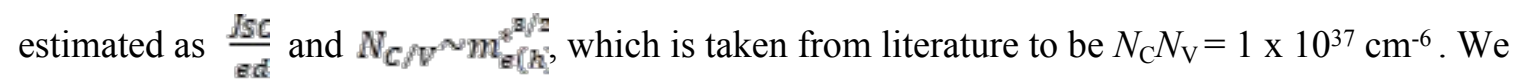
measure $\sim 450 \mathrm{~nm}$ thick perovskite layers and take a bandgap of $\sim 1.6 \mathrm{eV}$ (half-maximum).

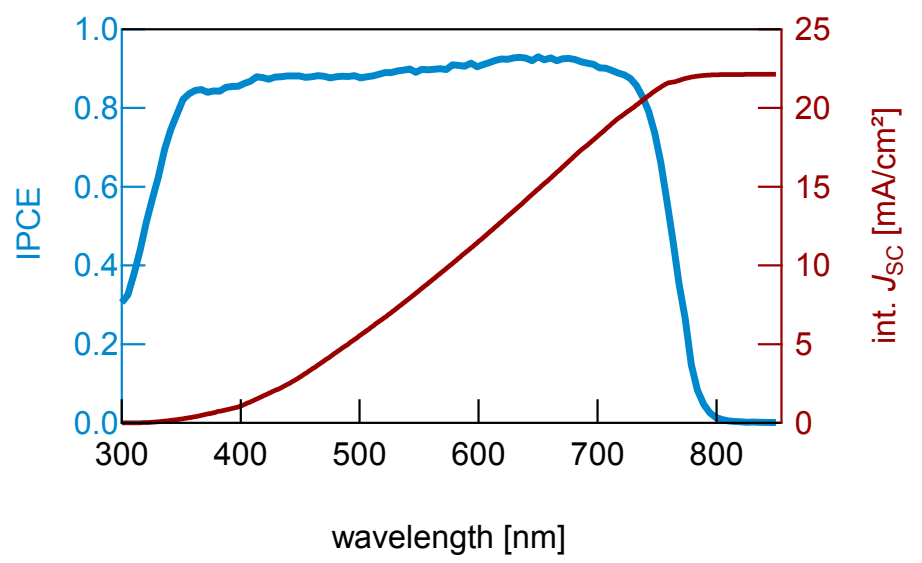

Figure S1. $E Q E_{\mathrm{PV}}$ spectrum and integrated $J_{\mathrm{SC}}$ for one of the $\mathrm{I}_{-} \mathrm{PFC}_{10}$ devices, delivering $22.1 \mathrm{~mA} / \mathrm{cm}^{2}$. 

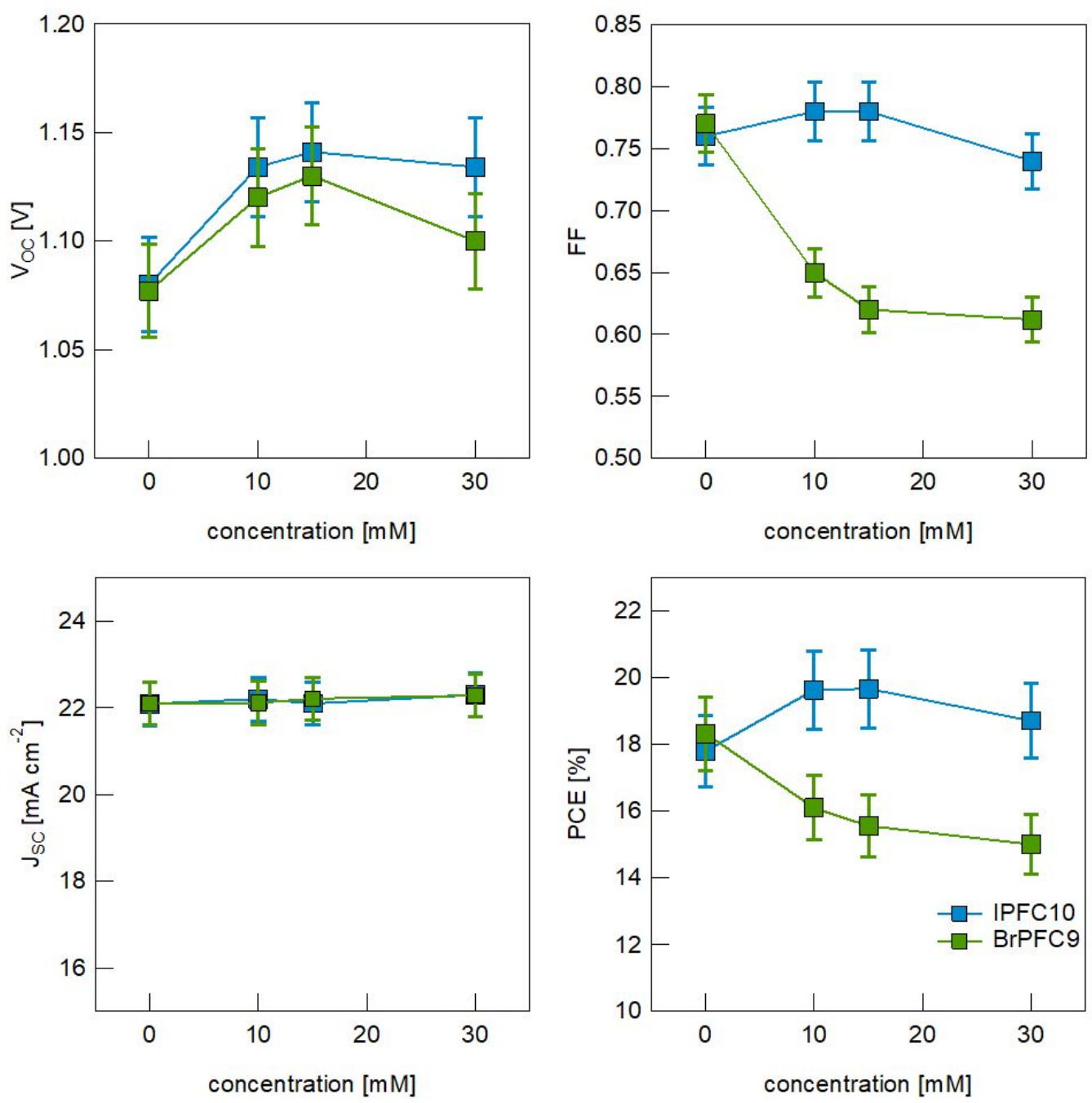

Figure S2. Concentration Optimization. Here we show preliminary optimization tests w.r.t concentration, i.e. PV parameters vs concentration of the SAM solution for IPFC10 and BrPFC9. 
a)

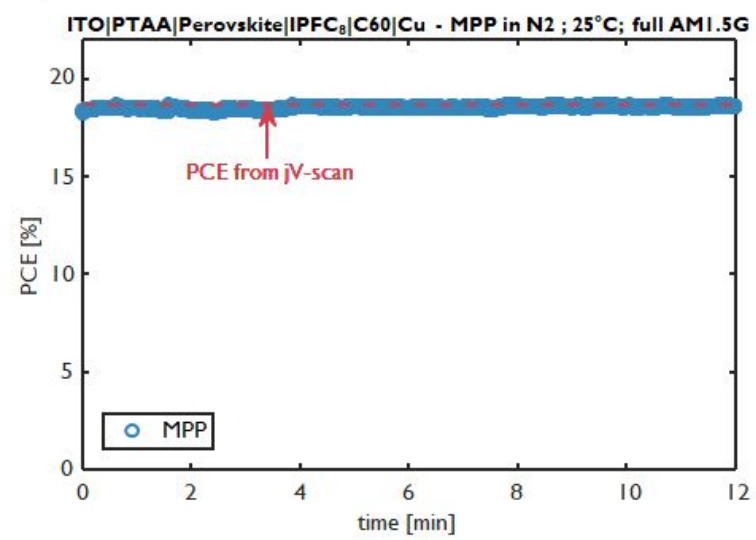

b)

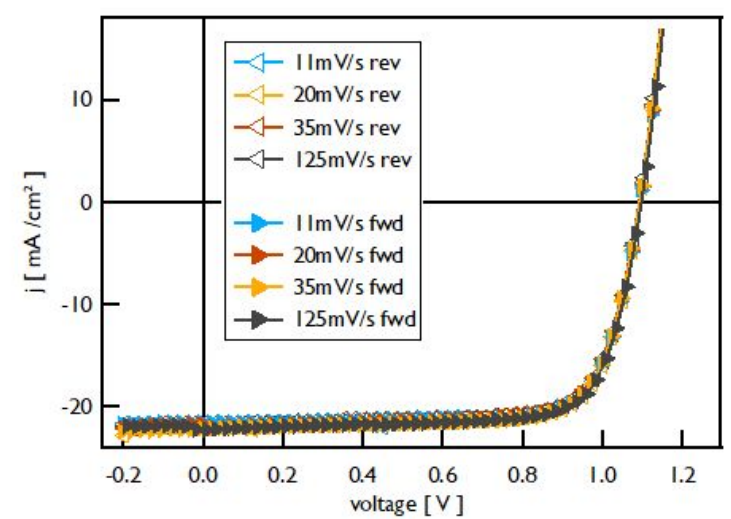

Figure S3. a) A representative solar cell at the lower end of the efficiency distribution under MPP tracking on a sun (blue) with the efficiency obtained from the $j V$ scan depicted in red. b) Hysteresis scan for the cell from a) in forward and reverse direction at different scan speeds showing the absence of hysteresis.

A

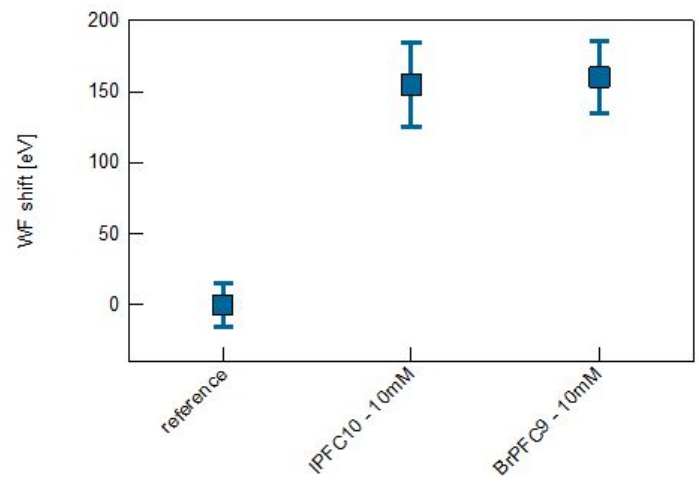

B

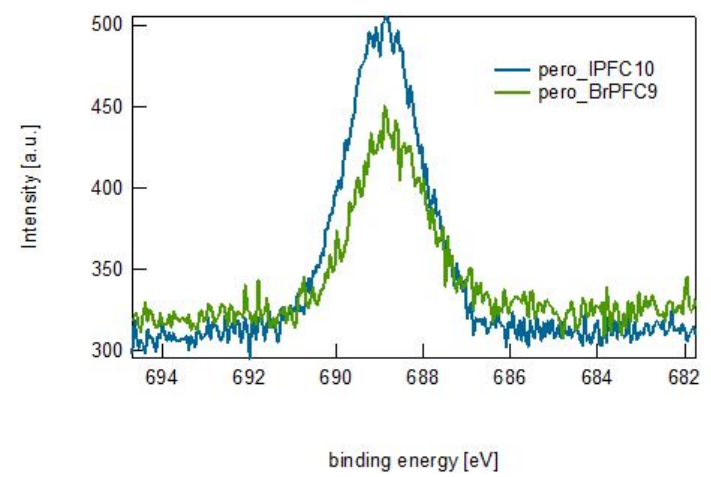

Figure S4. Photoemission and Kelvin Probe Study. (a) perovskite work function shift in nitrogen and in air for IPFC10 and BrPFC9. (b) F1s core-level spectra, showing the presence of fluorinated species on the perovskite surface. 
A
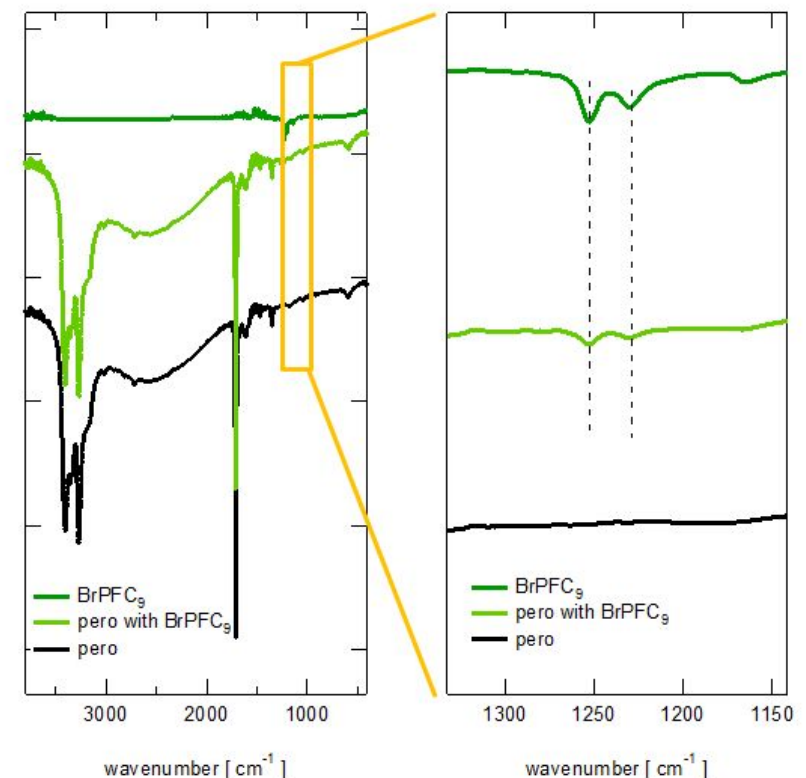

C

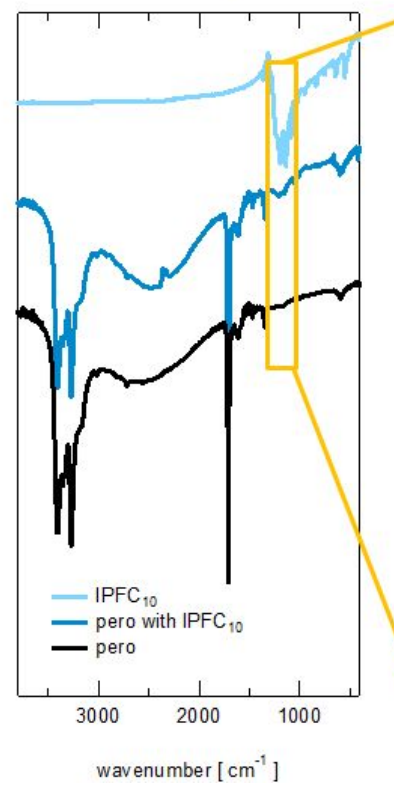

wavenumber $\left[\mathrm{cm}^{-1}\right]$

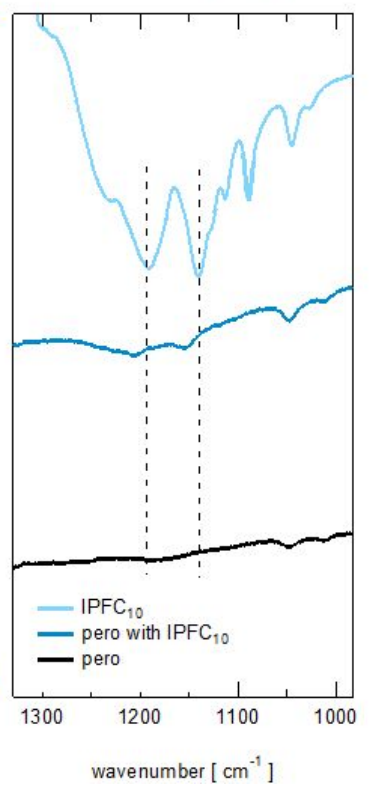

B

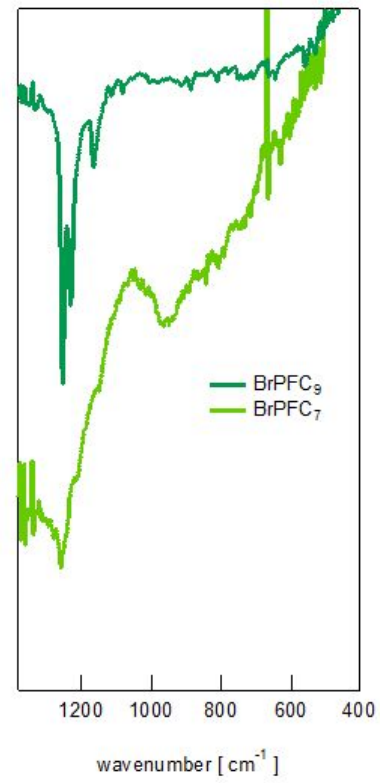

D

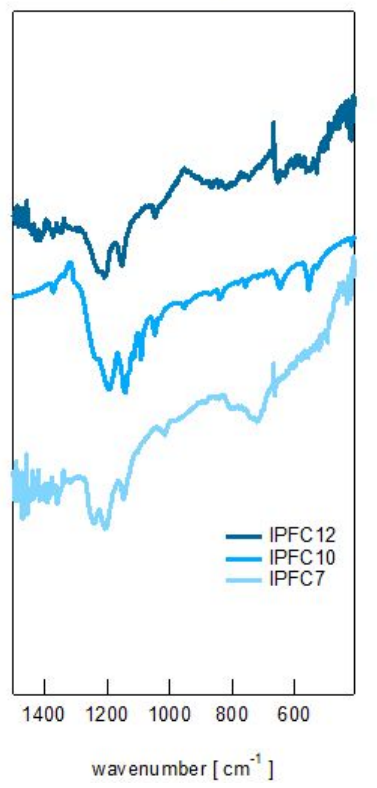

Figure S5. FTIR spectra. (A) BrPFC9 (dark green), perovskite (black) and perovskite with BrPFC9; no shift is visible. (B) comparison between BrPFC9 and BrPFC7 on Al2O3/A1 substrates. (C) IPFC10 (light blue), perovskite (black) and perovskite with IPFC10 (dark blue); the two visible peaks at $\sim 1200$ and $1150 \mathrm{~cm}-1$ shift, suggest of a small change in the electronic environment of the molecule if in contact with the perovskite. (D) comparison between IPFC7, 10 and 12 on Al2O3/Al substrates. 


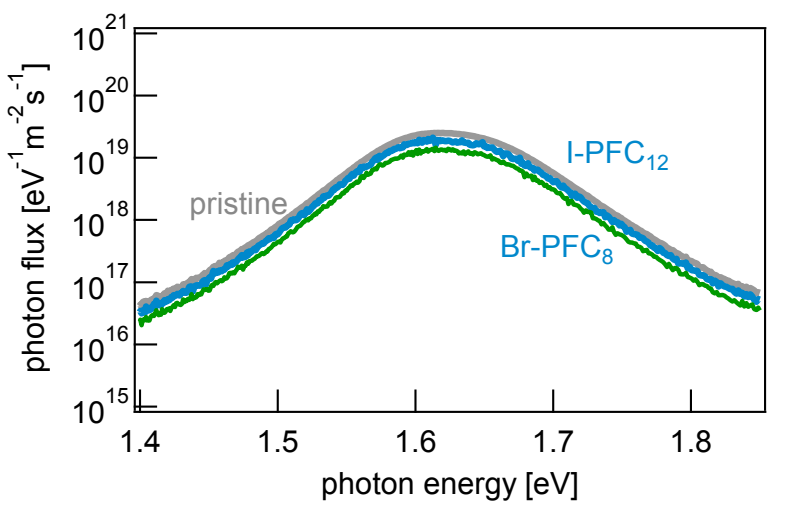

Figure S6. Absolute PL spectra of the perovskite with and without the SAMs, showing a negligible effect on the $P L Q Y$.

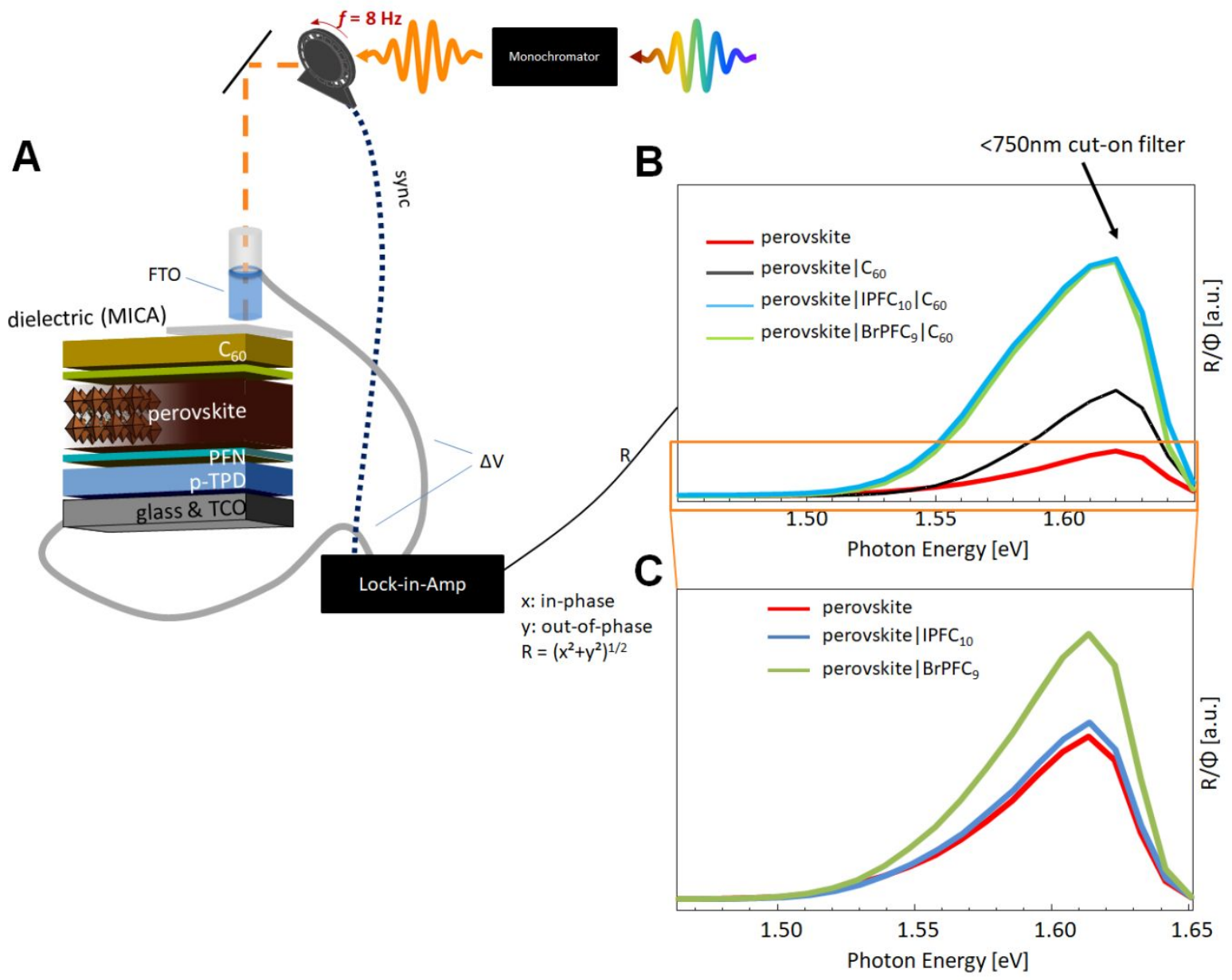

Figure S7. SPV. a) displays the experimental setup where a half-finished cell is illuminated with a small light perturbation and the corresponding change in surface photovoltage (due to electron accumulation at the top surface) is amplified (lock-in) and evaluated as a function of wavelength or photon energy. b) The corresponding spectra show, that in the case of a perovskite only (red) the signal at the band-edge is weakest, while it increases $\sim 2 \mathrm{x}$ if $\mathrm{C}_{60}$ as selective contact is present. If $\mathrm{IPFC}_{10}$ or $\mathrm{BrPFC}_{9}$ is interjected, the signal is even stronger, indicating the greater ability of electrons to accumulate in the $\mathrm{C}_{60}$ due to a reduction in recombination. c) without $\mathrm{C}_{60}$ the molecules slightly enhance the ability of the perovskite to accumulate electrons at the top surface. 


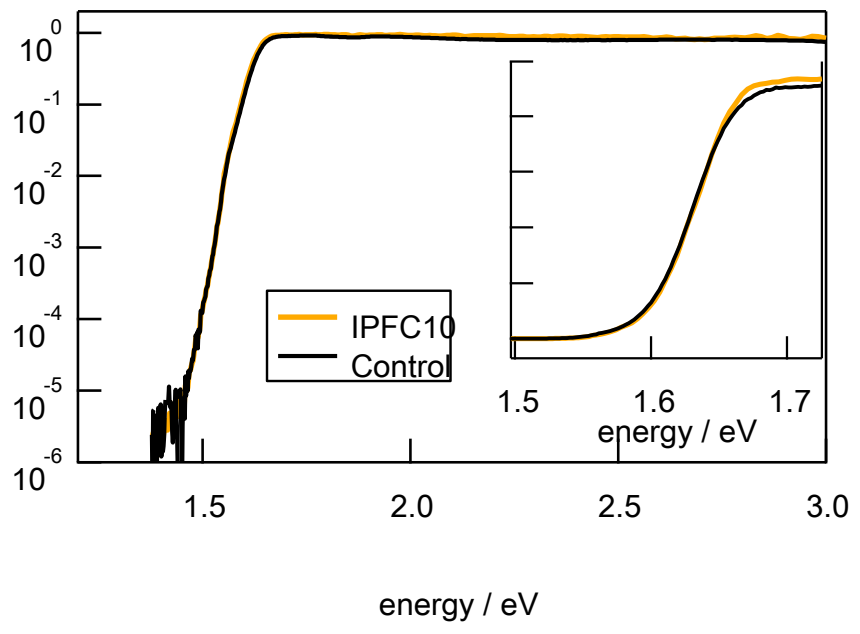

Figure S8. EQE spectra of solar cells with and without the I-PFC ${ }_{10}$ modification. The inset shows the linear plot displaying no change in bandgap.
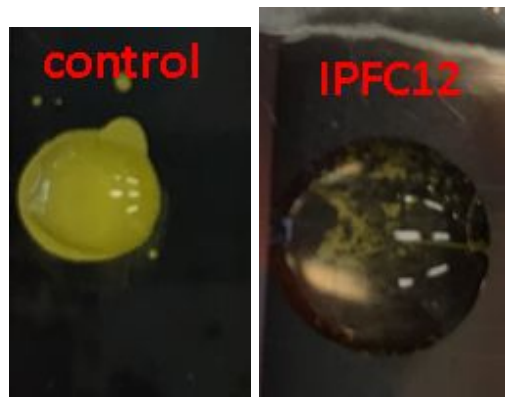

Figure S9. Photograph of perovskite solar cells without and with the SAM modification shortly $(<1$ min) after the drop of a water droplet (not the same amount). The modified perovskite surface mitigates water ingress as evident by the much less pronounced transformation to $\mathrm{PbI}_{2}$ (yellow). 

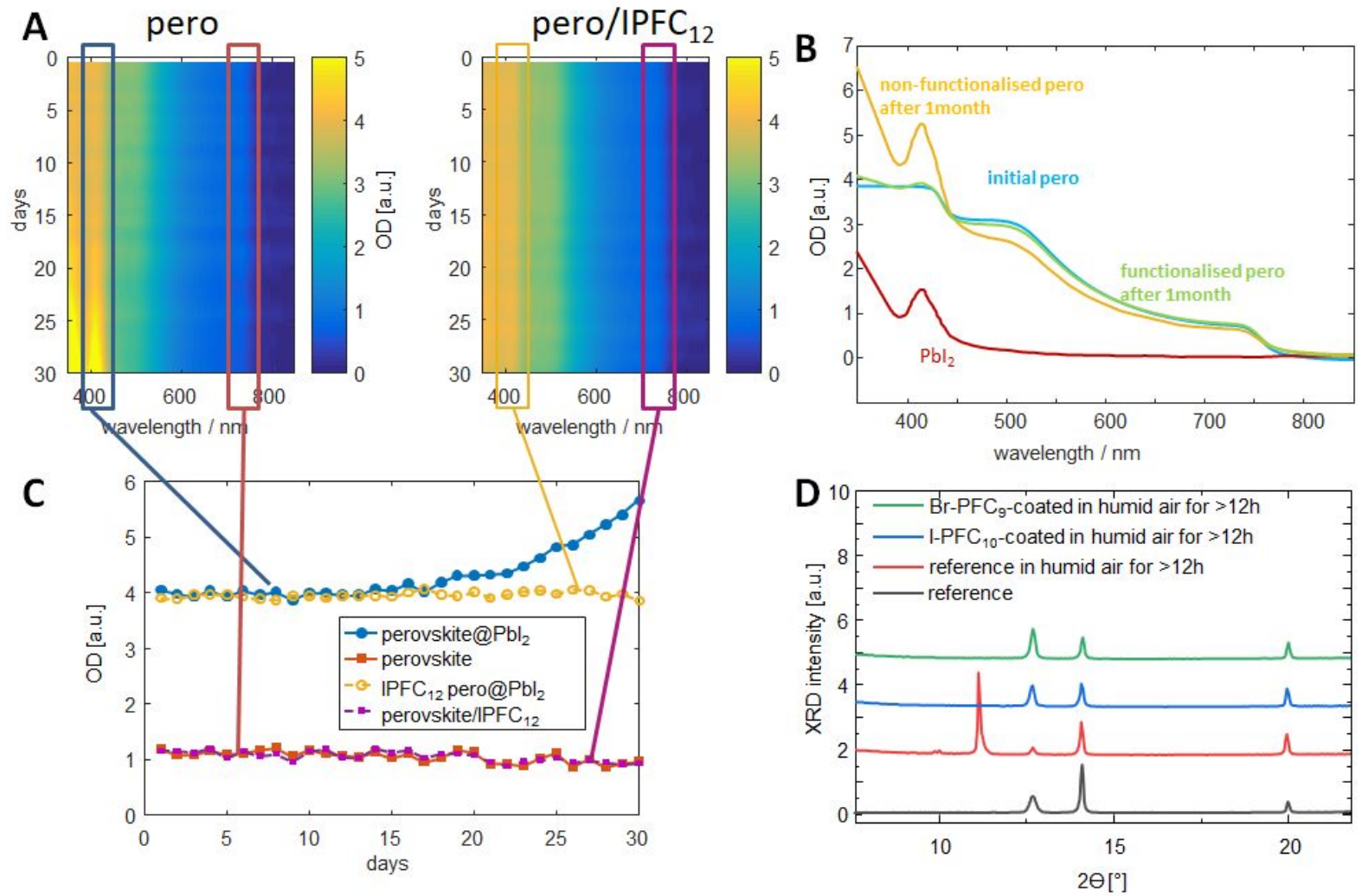

Figure S10. a) absorption spectra (spectra were taken in irregular time intervals and interpolated) over time of perovskite samples with (right) and without $\mathrm{I}_{-\mathrm{PFC}_{12}}$ (left) stored in air without encapsulation b) absorption of $\mathrm{PbI}_{2}$, the two samples before and after storage and c) the time-dependent evolution of characteristic spectral features (perovskite absorption near the band edge vs. a putative $\mathrm{PbI}_{2}$ signal which is clearly evolving in the case of a non-modified surface). d) presents XRD diffractograms (Bragg-Brentano configuration) of modified $\left(\mathrm{BrPFC}_{9}\right.$ - green, $\mathrm{IPFC}_{10}$ - blue) and unmodified (red) perovskite samples after humidity exposure as well as a reference (grey). The unmodified perovskite exhibits new peaks at $10^{\circ}$ and $11.5^{\circ}$ corresponding to hydrated lead species. These signals are absent in the case of the modified samples, supporting the observation of higher humidity resilience. 

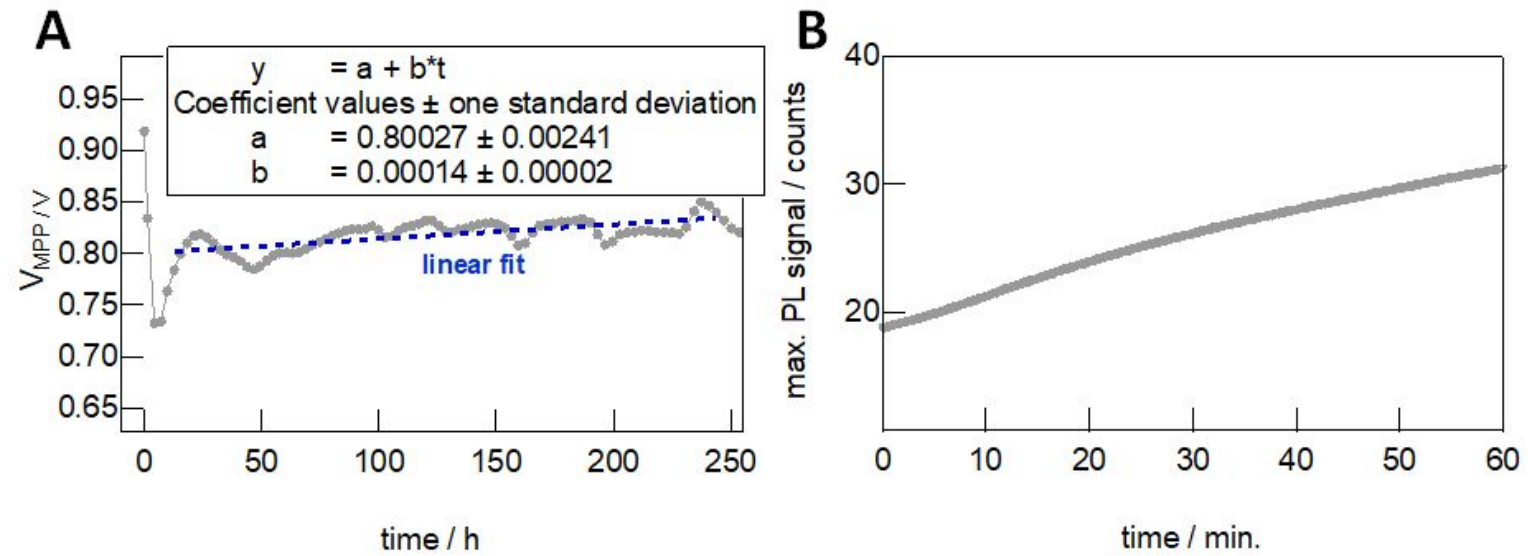

Figure S11. a) $V_{\text {MPP }}$ corresponding to $P_{\text {MPP }}$ in Figure 4e. b) peak PL intensity over the course of 1 hour in $\mathrm{N}_{2}$ with $\sim 1$ sun equivalent $445 \mathrm{~nm}$ light, showing an $\sim 1.5 \mathrm{x}$ increase over time.

\section{Additional References:}

(1) Perdew, J. P.; Burke, K.; Ernzerhof, M. Generalized Gradient Approximation Made Simple. Phys. Rev. Lett. 1996, 77, 3865-3868.

(2) Sabatini, R.; Gorni, T.; de Gironcoli, S. Nonlocal van Der Waals Density Functional Made Simple and Efficient. Phys. Rev. B 2013, 87, 041108.

(3) Monkhorst, H. J.; Pack, J. D. Special Points for Brillouin-Zone Integrations. Phys. Rev. B 1976, $13,5188-5192$.

(4) Giannozzi, P.; Baroni, S.; Bonini, N.; Calandra, M.; Car, R.; Cavazzoni, C.; Ceresoli, D.; Chiarotti, G. L.; Cococcioni, M.; Dabo, I.; Dal Corso, A.; de Gironcoli, S.; Fabris, S.; Fratesi, G.; Gebauer, R.; Gerstmann, U.; Gougoussis, C.; Kokalj, A.; Lazzeri, M.; et al. QUANTUM ESPRESSO: A Modular and Open-Source Software Project for Quantum Simulations of Materials. J. Phys. Condens. Matter 2009, 21, 395502. 